\title{
Effects of Resistive Exercise on Cerebral Blood Flow Velocity and Pulsatility Index of Healthy People
}

\author{
Jinhwa Jung, PhD, OT ${ }^{1)}$, Hyungkyu Kang, MSc, PT ${ }^{2)}$, Sunhwa Shim, MSc, OT ${ }^{3)}$, \\ Kinun Cho, MSc, $\mathrm{PT}^{4)}$, JAEHO Yu, PhD, $\mathrm{PT}^{5)}$ \\ 1) Department of Occupational Therapy, Semyung University \\ 2) Department of Physical Therapy, Sahmyook University \\ 3) Department of Occupational Therapy, Yonsei University \\ 4) Department of Physical Therapy, Seoul BukBu Hospital \\ 5) Department of Physical Therapy, Kangwon National University: 3 Hwangjo-ri, Dogye-eup, \\ Samcheok-si, Gangwon-do, 245-907, Republic of Korea. \\ TEL: +82 33-540-3370,FAX: +82 33-540-3379,E-mail:naresa@empal.com
}

\begin{abstract}
Purpose] This study compared the cerebral blood flow velocity and pulsatility index different exercise intensities before and after resistive exercises to evaluate the benefits of regular resistive exercise for the prevention of brain injuries such as stroke. [Subjects] Twelve healthy male adults were recruited for this study. [Methods] Transcranial Doppler ultrasonography was used to measure the cerebral blood flow velocity and pulsatility index. The test was conducted in 4 stages: at rest, moderate exercise, peak exercise, and recovery. After the pretest, each subject was given a 1-hour resistive exercise to be performed three times a week for 12 weeks, following which, a posttest was performed, and the resulting data were analyzed. [Results] Cerebral blood flow velocity at all stages, except systolic recovery, showed significant reductions after the intervention. The pulsatility index did not differ significantly at any stage. [Conclusion] Resistive exercise reduced cerebral blood flow velocity; therefore changes in cerebral blood flow should be monitored for the prevention of cerebrovascular diseases.

Key words: Resistive exercise, Cerebral blood flow velocity, Pulsatility index
\end{abstract}

(This article was submitted Apr. 17, 2012, and was accepted May 15, 2012)

\section{INTRODUCTION}

Traditionally, resistive exercise (RE) has been used in rehabilitation programs to increase the strength of patients. $\mathrm{RE}$ is effective for problems related to physical function or structure, such as changes caused by pain or weakness ${ }^{1)}$. In addition, RE increases energy costs and metabolism, thereby affecting hemodynamics ${ }^{2}$. Regular RE effectively eliminates foreign material in the arteries by reducing low-density lipoprotein cholesterol (LDL) levels while increasing highdensity lipoprotein (HDL) cholesterol levels ${ }^{3)}$.

Generally, the presence of cerebral artery stenosis results in high cerebral blood flow velocity (CBFV), and the severity of this condition is a pre-disposing factor for stroke ${ }^{4}$. Patients with cerebral artery stenosis initially receive drug treatment; however, severe hemodynamic disorder requires surgery. Hence, prevention of stroke through the modification of risk factors is very important $t^{5}$.

Previous studies have showen that RE of the upper extremities temporarily increases the CBFV of the middle cerebral artery $(\mathrm{MCA})^{6}$ ); the rise is gradual during exercise but it subsequently drops from $60 \%$ of maximal heart rate $\left(\mathrm{HR}_{\max }\right)^{7)}$. Querido and Sheel reported that brain oxidation in individuals exercising regularly is not a limiting factor of physical, activity because the cerebral cortex is well protected even during exercise ${ }^{8)}$. However, individuals with sedentary lifestyles are more prone to brain damage during or after high intensity exercise.

Previous studies have reported that $\mathrm{CBFV}$ increase is proportional to strength, which can be improved through various exercises ${ }^{6)}$. In addition, a comparison between elderly and young individuals showed that vasoconstriction increases with age, which consequently increases CBFV. However, these studies were mostly conducted as cross sectional studies designed to detect rapid changes in hemodynamics; and the long-term effects of RE on CBFV have not yet been assessed. Further, while changes in CBFV resulting from aerobic exercises have been investigated, similar studies involving RE are rare.

The pulsatility index (PI) reflects the downstream resistance to blood flow caused by vasoconstriction. There is a need to demonstrate the change of PI according to RE. Hence, to verify the benefits of regular RE for the prevention of brain injuries such as stroke, we assessed its effects on CBFV and PI of healthy subjects who exercised according to the exercise intensity healthy subjects for 12 weeks. We hypothesized that RE would affect CBFV and PI. 
Table 1. Comparison of cerebral blood flow velocity and pulsatility index

\begin{tabular}{cccccc}
\hline \multicolumn{2}{c}{ Item } & At rest & Moderate exercise & Peak exercise & Recovery \\
\hline SBFV & Pretest & $116.9 \pm 14.2$ & $160.7 \pm 27.6$ & $159.0 \pm 35.5$ & $137.1 \pm 24.9$ \\
$(\mathrm{~mm} / \mathrm{sec})$ & Posttest & $91.6 \pm 10.2^{* *}$ & $140.7 \pm 22.3^{*}$ & $129.5 \pm 23.4^{*}$ & $118.5 \pm 21.5$ \\
$\mathrm{DBFV}$ & Pretest & $52.9 \pm 9.8$ & $63.9 \pm 15.0$ & $49.2 \pm 11.6$ & $49.3 \pm 15.9$ \\
$(\mathrm{~mm} / \mathrm{sec})$ & Posttest & $41.4 \pm 3.4^{* *}$ & $54.5 \pm 11.2^{*}$ & $36.5 \pm 8.9^{* *}$ & $36.3 \pm 5.2^{* *}$ \\
$\mathrm{MBFV}$ & Pretest & $75.7 \pm 11.0$ & $100.9 \pm 22.8$ & $93.5 \pm 19.8$ & $76.8 \pm 14.7$ \\
$(\mathrm{~mm} / \mathrm{sec})$ & Posttest & $60.9 \pm 6.4^{* *}$ & $85.7 \pm 14.4^{*}$ & $71.5 \pm 12.3^{* *}$ & $61.0 \pm 6.8^{* *}$ \\
PI & Pretest & $0.8 \pm 0.1$ & $0.9 \pm 0.1$ & $1.1 \pm 0.1$ & $1.1 \pm 0.2$ \\
$(\mathrm{score})$ & Posttest & $0.8 \pm 0.1$ & $1.0 \pm 0.2$ & $1.3 \pm 0.2$ & $1.3 \pm 0.2$ \\
\hline
\end{tabular}

Note. All values are mean \pm standard deviation. SBFV, Systolic blood flow velocity; DBFV, Diastolic blood flow velocity; MBFV, Mean blood flow velocity; PI, Pulsatility index. ${ }^{*} \mathrm{p}<0.05,{ }^{* *} \mathrm{p}<0.01$.

\section{SUBJECTS AND METHODS}

This study had a one group pre-posttest design. The study subjects were 12 healthy male students attending the University of Korea, who participated voluntarily and understood the study contents. To exclude the effects of pre-existing conditions on $\mathrm{CBFV}$, we selected subjects who had regularly exercised for 1 year and had no history of diabetes, cerebral artery or coronary artery diseases. The objective and procedure of the study were explained to all the participants who then signed a participation consent form. The average age, height and weight of the participants was $21.42 \pm 1.44$ years (mean \pm standard deviation); $175.33 \pm 5.06 \mathrm{~cm}$, and $70.50 \pm 3.98 \mathrm{~kg}$, respectively. Height and weight were measured using a body composition analyzer (InBody 3.0, Biospace, Korea). After the pretest, each participant performed a 1-hour RE, 3 times a week for 12 weeks, following which, the posttest was performed.

A 10-minute warm up exercise consisting of treadmill walking and stretching was conducted before and after RE. RE consisted of 8 programs including, leg extension, leg curl, leg press, heel raise, bench press, overhead pull down, arm curl, and arm extension. The subjects performed 3 sets of 12 repetitions at the $70 \%$ level of individual maximal resistance, with a resting time between each trial of 30 seconds.

During the pre and posttests, Bruce's protocol was used to control the exercise intensity, and the velocity and grade of the treadmill were gradually increased every 3 minutes ${ }^{9)}$. Transcranial Doppler (TCD) ultrasonography was used to measure CBFV and PI (Power M-mode 150, Spencer technologies, USA). The test was conducted a total of 4 times (at rest, moderate exercise, peak exercise, and recovery). During the period of exercise, moderate exercise was performed at $60 \%$ of $\mathrm{HR}_{\max }$ and peak exercise was performed at $100 \%$ $\mathrm{HR}_{\max }$ based on maximal heart rate (220-age). The recovery period was chosen as 2 minutes after exercise termination. As the participants completed each stage of the exercise, they were moved to a bed adjacent to the treadmill, and TCD ultrasonography was performed in the supine position. A transtemporal approach was used to measure blood flow in the MCA using a 50-64 mm deep probe connected to the body. After identifying the most active location of resistance in MCA, systolic, diastolic, and mean CBFV, and PI were measured and the location of MCA was marked as a $0.5 \mathrm{~cm}$ radius circle with a marker pen during the initial test for the next stage's test. For TCD measurement, the participants were asked to lie on a bed with assistance from 3 examiners, and measurements were recorded within 20 minutes, after which the participants returned to the treadmill.

Statistical analysis of the differences in systolic, diastolic, and mean $\mathrm{CBFV}$, and PI before and after the RE intervention using the paired t-test. The independent variable of this study was RE and the dependent variables were CBFV, and PI. Statistical significance was accepted for values of $p<$ 0.05 . SPSS Version 12.0 was used to calculate the mean and standard deviation.

\section{RESULTS}

Changes in systolic, diastolic, and mean CBFV, and PI before and after the RE intervention, are shown in Table 1. After the RE intervention, CBFV at all stages except systolic recovery was significantly reduced $(p<0.05)$. PI did not differ significantly at any stage.

\section{DISCUSSION}

The aim of this study was to evaluate the effects of RE on CBFV and PI. RE is an effective exercise method which increases strength, endurance, and balance, because exercise equipment and the body of a physical therapist create physical resistance for the subjects. RE has been widely used in physical therapy and rehabilitation programs for patients with musculoskeletal and neurologic disorders ${ }^{10)}$. Regular RE improves metabolism by increasing muscular volume and lipolysis and has favorable effects on endothelial function and major cardiovascular adaptations through the removal of thrombus, and LDL cholesterol in blood vessels as measured by the increase of high density lipoprotein cholesterol ${ }^{11,12)}$. However, studies of blood vessel health improvement have rarely been performed owing to the difficulties associated with long-term studies, as compared to studies of aerobic exercises.

In general, CBFV increase gradually until $60 \%$ of $\mathrm{HR}_{\max }$, compared to at rest, but decreased thereafter; these hemodynamic changes are seen in healthy subjects of all ages ${ }^{13)}$. Therefore, the moderate exercise stage of in this study was 
chosen as $60 \%$ of $\mathrm{HR}_{\max }$, and results of previous studies as well as effects of current study according to exercise intensity on changes in $\mathrm{CBFV}$ were consistent pre and post intervention.

In our study, CBFV decreased after the RE intervention at all stages, except systolic recovery. Sohemaker et al. reported that the mean blood flow velocity of cardiac and skeletal muscles was reduced by $12 \%$ after a 9 -week isometric exercise period but not $\mathrm{CBFV}^{14)}$. In addition, Ivey et al. conducted a human study and reported that a 6-month exercise regime for patients with stroke reduced CBFV of $\mathrm{MCA}^{15)}$, similar to the results of a previous study using aerobic exercises. Therefore, RE is effective at reducing $\mathrm{CBFV}$, and as a consequence, at preventing cerebrovascular diseases, if practiced as regularly as aerobic exercises. RE alters plasma levels of brain-derived neurotrophic factor $(\mathrm{BDNF})^{16)}$. Additionally, RE represents a mode of behavioral intervention which enhances brain health by increasing levels of BDNF and promoting neurogenesis ${ }^{17)}$.

PI reflects the downstream resistance to blood flow caused by vasoconstriction. In previous studies, PI was used as an effective indicator of changes in peripheral vascular resistance following exercise ${ }^{18)}$. The results of our study show that PI did not differ significantly at any stage of exercise. PI is defined as the difference in systolic and diastolic CBFV, divided by mean CBFV over the cardiac cycle. In our study, CBFV was reduced after the RE intervention in all subject; therefore. the change in PI may have been too small to detect. Vascular resistance is associated with waste materials such as thrombus in the vessels and vasoconstriction. Therefore, in our study, the intervention may have reduced waste in the vessels. Our study was limited by the choice of healthy adults as participants; patients with cerebrovascular diseases were not represented. Hence, further studies involving patients with cerebrovascular disease are warranted. Also, there is a need to investigate the longterm effects of RE and compare them with aerobic exercise.

In conclusion, given that $\mathrm{RE}$ reduces $\mathrm{CBFV}$ and, awareness of CBFV changes is important for timely prevention of cerebrovascular diseases, monitoring of CBFV in the field of physical therapy is required.

\section{REFERENCES}

1) O'Brien K, Tynan AM, Nixon S, et al.: Effects of progressive resistive exercise in adults living with HIV/AIDS: systematic review and metaanalysis of randomized trials. AIDS Care, 2008, 20: 631-653. [Medline] [CrossRef]

2) Caruso JF, Borgsmiller JA, Riner RD, et al.: Net energy expenditure of gravity-independent high-speed resistive exercise done by women. Aviat Space Environ Med, 2012, 83: 111-117. [Medline] [CrossRef]

3) Hurley BF: Effects of resistive training on lipoprotein-lipid profiles: a comparison to aerobic exercise training. Med Sci Sports Exerc, 1989, 21: 689-693. [Medline]

4) Hunter AJ, Snodgrass SJ, Quain D, et al.: HOBOE (Head-of-Bed Optimization of Elevation) Study: association of higher angle with reduced cerebral blood flow velocity in acute ischemic stroke. Phys Ther, 2011, 91: 1503-1512. [Medline] [CrossRef]

5) Vernieri F, Pasqualetti P, Passarelli F, et al.: Outcome of carotid artery occlusion is predicted by cerebrovascular reactivity. Stroke, 1999, 30: 593-598. [Medline] [CrossRef]

6) Miyazawa $\mathrm{T}$, Horiuchi M, Ichikawa D, et al.: Kinetics of exercise-induced neural activation; interpretive dilemma of altered cerebral perfusion. Exp Physiol, 2012, 97: 219-227. [Medline]

7) Hiura M, Kinoshita N, Izumi S, et al.: Comparison of the kinetics of pulmonary oxygen uptake and middle cerebral artery blood flow velocity during cycling exercise. Adv Exp Med Biol, 2012, 737: 25-31. [Medline] [CrossRef]

8) Querido JS, Sheel AW: Regulation of cerebral blood flow during exercise. Sports Med, 2007, 37: 765-782. [Medline] [CrossRef]

9) Bruce RA, Blackmon JR, Jones JW, et al.: Exercising testing in adult normal subjects and cardiac patients. Pediatrics, 1963, 32: 742-756. [Medline]

10) Polito MD, da Nobrega AC, Farinatti P: Blood pressure and forearm blood flow after multiple sets of a resistive exercise for the lower limbs. Blood Press Monit, 2011, 16: 180-185. [Medline] [CrossRef]

11) Selig SE, Carey MF, Menzies DG, et al.: Moderate-intensity resistance exercise training in patients with chronic heart failure improves strength, endurance, heart rate variability, and forearm blood flow. J Card Fail, 2004, 10: 21-30. [Medline] [CrossRef]

12) Rakobowchuk M, McGowan CL, de Groot PC, et al.: Endothelial function of young healthy males following whole body resistance training. J Appl Physiol, 2005, 98: 2185-2190. [Medline] [CrossRef]

13) Seifert $T$, Secher NH: Sympathetic influence on cerebral blood flow and metabolism during exercise in humans. Prog Neurobiol, 2011, 95: 406-426. [Medline] [CrossRef]

14) Shoemaker JK, Pozeg ZI, Hughson RL: Forearm blood flow by Doppler ultrasound during test and exercise: tests of day-to-day repeatability. Med Sci Sports Exerc, 1996, 28: 1144-1149. [Medline] [CrossRef]

15) Ivey FM, Ryan AS, Hafer-Macko CE, et al.: Improved cerebral vasomotor reactivity after exercise training in hemiparetic stroke survivors. Stroke, 2011, 42: 1994-2000. [Medline] [CrossRef]

16) Schiffer T, Schulte S, Hollmann W, et al.: Effects of strength and endurance training on brain-derived neurotrophic factor and insulin-like growth factor 1 in humans. Horm Metab Res, 2009, 41: 250-254. [Medline] [CrossRef]

17) Cotman CW, Berchtold NC, Christie LA: Exercise builds brain health: key roles of growth factor cascades and inflammation. Trends Neurosci, 2007, 30: 464-472. [Medline] [CrossRef]

18) Osada T: Exercise-related time course of pulsatility index in brachial artery following forearm exercise assessed by Doppler ultrasound. Tohoku J Exp Med, 2004, 203: 241-252. [Medline] [CrossRef] 